\title{
Saúde mental das crianças e adolescentes em tempos de pandemia: uma revisão narrativa
}

\author{
Mental health of children and adolescents in times of pandemic: a narrative review \\ La salud mental de los niños y adolescentes en tiempos de pandemia: una revisión \\ narrativa
}

Liane Franco Barros Mangueira ${ }^{1 *}$, Ricardo Andre Medeiros Negreiros ${ }^{1}$, Margareth de Fátima Formiga Melo Diniz ${ }^{1}$, José Kenio de Sousa ${ }^{1}$.

\section{RESUMO}

Objetivo: Realizar uma revisão narrativa acerca dos impactos na saúde mental de crianças e adolescentes durante períodos de pandemia. Revisão bibliográfica: Foram analisadas as múltiplas consequências para a saúde mental infantojuvenil, avaliando-se os aspectos relacionados a doença em questão e suas repercussões psicossomáticas desencadeadas ou intensificadas em momentos excepcionais de isolamento social. Verificou-se prevalência do medo em perder familiares ou de adoecimento, ao ponto de desencadear pensamentos negativos constantes. Diversos acontecimentos durante a pandemia podem ser traumáticos para esta faixa etária, sejam o confinamento a pequenos espaços, a falta de interação social ou a ameaça da doença, podendo gerar problemas agudos ou crônicos. Considerações finais: As pandemias estão associadas as mais diversas implicações na vida de crianças e adolescentes, podendo ser observadas sensações de medo, tédio e solidão, além de alterações dos padrões de sono, alimentação e comportamento. Portanto, é fundamental que a família assuma um papel de proteção e cuidado, desenvolvendo atividades lúdicas e práticas para aliviar o estresse, dentro de um ambiente seguro e acolhedor, respeitando os limites de uma rotina saudável e contribuindo para a preservação do bem-estar e apoio mútuo dentro da coletividade.

Palavras-chave: Cuidado da criança, Bem-estar da criança, Defesa da criança e do adolescente, Saúde mental, Pandemias.

\begin{abstract}
Objective: Conduct a narrative review on the impacts on the mental health of children and adolescents during periods of pandemic. Bibliographical review: The multiple consequences for children's and adolescent's mental health were analysed, evaluating the aspects related to the disease in question and its psychosomatic repercussions triggered or intensified in exceptional moments of social isolation. There was a prevalence of fear of losing family members or of becoming ill, to the point of triggering constant negative thoughts. Several events during the pandemic can be traumatic for this age group, be it confinement to small spaces, lack of social interaction or the threat of the disease, and can generate acute or chronic problems. Final considerations: Pandemics are associated with the most diverse implications in the lives of children and adolescents, and feelings of fear, boredom and loneliness can be observed, as well as changes in sleep, eating and behaviour patterns. Therefore, it is fundamental that the family assumes a role of protection and care, developing playful and practical activities to relieve stress, within a safe and welcoming environment, respecting the limits of a healthy routine and contributing to the preservation of well-being and mutual support within the community.
\end{abstract}

Keywords: Child care, Child welfare, Child advocacy, Mental health, Pandemics.

\footnotetext{
1 Universidade Federal da Paraíba (UFPB), João Pessoa - PB.

*E-mail: liane.franco@terra.com.br
} 


\section{RESUMEN}

Objetivo: Realizar un examen narrativo de los efectos de los niños y adolescentes en la salud mental durante los períodos de pandemia. Revisión bibliográfica: Se analizaron las múltiples consecuencias para la salud mental de los niños y adolescentes, evaluando los aspectos relacionados con la enfermedad en cuestión y sus repercusiones psicosomáticas desencadenadas o intensificadas en momentos excepcionales de aislamiento social. Se daba una prevalencia del miedo a perder a los miembros de la familia o a la enfermedad, hasta el punto de desencadenar constantes pensamientos negativos. Varios acontecimientos durante la pandemia pueden ser traumáticos para este grupo de edad, como el confinamiento en espacios reducidos, la falta de interacción social o la amenaza de la enfermedad, y pueden generar problemas agudos o crónicos. Consideraciones finales: Las pandemias se asocian con las más diversas implicaciones en la vida de los niños y adolescentes, y se pueden observar sentimientos de miedo, aburrimiento y soledad, así como cambios en los patrones de sueño, alimentación y comportamiento. Por lo tanto, es fundamental que la familia asuma un papel de protección y cuidado, desarrollando actividades lúdicas y prácticas para aliviar el estrés, dentro de un entorno seguro y acogedor, respetando los límites de una rutina saludable y contribuyendo a la preservación del bienestar y el apoyo mutuo dentro de la comunidad.

Palabras clave: Cuidado del niño, Bienestar del niño, Defensa del niño, Salud mental, Pandemias.

\section{INTRODUÇÃO}

Em dezembro de 2019, após uma série de casos envolvendo doenças que causaram pneumonia e morte, inicialmente em Wuhan, na China, descobriu-se um agente similar ao coronavírus da síndrome respiratória aguda grave (SARS-CoV), responsável pelo surto viral em 2002. O novo vírus, que passou a ser conhecido como SARS-CoV-2, é o causador da doença do coronavírus 2019 (COVID-19), segundo a Organização Mundial de Saúde (OMS) (AHN DG, et al., 2020).

Após se espalhar por mais de 140 outros países, a doença foi declarada como uma questão global de saúde, ou seja, uma pandemia, no dia 11 de março de 2020, pela OMS. Essa afecção caracteriza-se por sintomas de febre, dor de garganta, fadiga, tosse e dispneia, podendo culminar em graves infecções no trato respiratório humano (ZHAI P, et al., 2020).

Pandemia é o termo usado para o escalonamento de determinada doença, memorando imagens de medo, desorganização e desagregação social. Os reflexos psicossociais nas populações afetadas são, muitas vezes, mais prejudiciais que a doença propriamente dita (PERRIN PC, et al., 2009). Associada a esses fatores, as crianças e adolescentes estão em risco de adoecerem ou presenciarem doentes os seus pais, relativos e outros membros significantes de suas comunidades (EARLS F, et al., 2008).

Sabe-se que, durante o processo de disseminação da COVID-19 pelo mundo, houve uma grande ruptura no cotidiano das pessoas, impactando em sua saúde física e mental. O aumento dos números de casos, a propagação por parte da mídia e os desfechos negativos ocasionados pela doença, contribuem para o aumento dos níveis de estresse da população (LIMA CKT, et al., 2020). As consequências danosas desse bombardeio de estressores impacta o desenvolvimento, o metabolismo e o comportamento do sujeito, com o risco potencial de transtornos mentais agudos e crônicos (CHROUSOS GP, 2009).

Com o pretexto do isolamento social, muitos serviços básicos, relacionados a educação, assistência a saúde e lazer, ficam inacessíveis, além de carecer de redes de suporte a saúde mental da criança e do adolescente. Estes, mantidos em casa, precisam reorganizar suas rotinas, fator que pode ter forte impacto em seus aspectos psicológicos e emocionais (FEGERT JM, et al., 2020).

Nesta experiência desconfortável de quarentena, os mais vulneráveis a danos psicológicos são as crianças, adolescentes, idosos e pessoas de grupos socioeconômicos mais baixos (USHER K, et al., 2020). Nas áreas urbanas, em especial, encontrou-se grande impacto nos níveis de depressão e ansiedade, majoritariamente no sexo feminino (ÖZDIN S e ÖZDIN SB, 2020). 
Desconhecer a causa, progressão da doença e seus resultados, bem como a desinformação e os rumores, podem gerar sentimento de insegurança e fazer com que as pessoas se sintam ansiosas (USHER K, et al., 2020). No contexto da pandemia, a ansiedade e o pânico atingem a muitos que escutam e acompanham informações sobre a doença, o que pode gerar desfechos de saúde negativos em caso de contração da mesma, quando comparado a indivíduos que não enfrentam o adoecimento psíquico (KELLY BD, 2020). Além do que, as mídias sociais por vezes contribuem para a dispersão do pavor e histeria na população em geral, especialmente nos jovens, podendo desencadear ansiedade psicopatológica (AHMAD AR, et al., 2020).

As situações incertas podem levar a alterações de comportamento, que vão desde ansiedade, insônia e frustração até hábitos compulsivos e obsessivos. Observa-se ainda que tais situações podem gerar desconfiança e fortalecer a crença em teorias conspiratórias, o que pode contribuir para menor adesão as recomendações em saúde, provocando um comportamento de recusa ao conhecimento científico (SHUJA $\mathrm{KH}$, et al., 2020).

As crianças e adolescentes, nas circunstâncias de crise, são especialmente susceptíveis a problemas de saúde mental, como observado durante outros momentos similares na história. Portanto, o planejamento estratégico em saúde para este grupo é essencial (STEVENSON E, et al., 2009).

Em tempos difíceis, a calma e a segurança entram em conflito, especialmente no contexto familiar. Manterse alerta para os riscos ao mesmo tempo em que se reafirma o ambiente seguro se torna um desafio quando na presença de crianças, pois elas percebem, assim como os pais, as contradições entre os atos e as palavras (NUNN K, 2020).

O objetivo principal deste estudo é explorar os efeitos da pandemia nas crianças e adolescentes, apresentando os aspectos referentes a saúde mental, observados em períodos vivenciados anteriormente, dada a importância de se planejar uma rede de apoio eficaz e fortalecer as ações de enfrentamento a problemática decorrente da COVID-19.

\section{REVISÃO BIBLIOGRÁFICA}

A pandemia da COVID-19 traz consigo diversos impactos, como o fechamento de escolas, impedimento as atividades presenciais e outras medidas que produzem reflexos sociais, psicológicos e econômicos. Gerenciar o dia a dia dos filhos, objetivando preservar a saúde mental dos jovens, amparando-os em suas aflições e sofrimentos, é uma atividade desenvolvida pelos pais já estafados devido as mudanças proporcionadas pelo contexto de saúde observado mundialmente (ALMEIDA RS, et al., 2020). Os pais de crianças deficientes, especialmente, acabam por lidar com sobrecarga de trabalho e estresse, visto que seus filhos estão mais propícios a contaminação pela dificuldade de implementar as normas sanitárias adequadas.

Além da possibilidade de reduzir os sentimentos de segurança, os eventos nocivos da pandemia têm a capacidade de relembrar o fato da finitude da vida e isso pode ocasionar efeitos adversos a saúde mental (ÖZDIN S e ÖZDIN SB, 2020). Tal fato evidencia a necessidade dos profissionais que atendem a saúde mental realizarem intervenções no sentido de abrandarem o medo e a desconfiança irracionais muitas vezes observados nas populações em pânico (SHUJA KH, et al., 2020).

\section{Literatura relacionada ao medo durante pandemias}

O medo é a resposta emocional a ameaças iminentes reais ou percebidas, usualmente acompanhado de expectativa apreensiva e desconforto (CROCQ MA, 2015). Também considerado um mecanismo adaptativo de defesa que envolve uma série de processos biológicos, o medo, durante uma pandemia, pode aumentar os níveis de ansiedade e estresse em indivíduos saudáveis, além de intensificar sintomas em pessoas com condições psiquiátricas anteriores (ORNELL F, et al., 2020).

Muitas vezes, este medo é exposto como consequência de ansiedade adjacente, sendo esta um sintoma desagradável compreendendo a apreensão, tensão, ou qualquer outro incômodo relativo ao inexplorado ou ao novo, com capacidade de comprometer o bem-estar e o desempenho sobre as atividades diárias do indivíduo. Há relatos na literatura demonstrando que existem diversos fatores da ansiedade associados a depressão, a exemplo de desequilíbrios químicos cerebrais, características de personalidade, vulnerabilidade genética e eventos situacionais (SCHMIDT DRC, et al., 2011). 
A suspeita ou confirmação da enfermidade, bem como as incertezas relacionadas a situação econômica mundial e nacional, são fontes de medo associadas ao processo de pandemia, com efeitos individuais que podem persistir mesmo após o surto (ONYEAKA HK, et al., 2020).

Dentre os artigos analisados, percebeu-se que todos citavam a vivência do medo por parte de crianças e adolescentes, sendo aquele de perder alguém da família o mais presente, em particular quando há alguém no grupo de risco, tornando-se mais frequente o aparecimento destes pensamentos (FEGERT JM, et al., 2020). O fato de estar em casa, devido ao isolamento social, mostrou a prevalência de frustração e tédio, além do medo da infecção propriamente dita (CUI Y, et al., 2020).

O temor de ficar doente também pode gerar consequências graves, como a somatização aguda. Durante a pandemia da COVID-19, há casos em que, mesmo após a confirmação da ausência do vírus via exames laboratoriais, o medo excessivo e desproporcional de sintomas permanece (COLIZZI M, et al., 2020), sendo este uma das principais manifestações que a criança e o adolescente experimentam após vivenciar ou presenciar o incidente biológico, acompanhados de outros sintomas que podem ser súbitos e elusivos (SHANNON MW, et al., 2006).

\section{Consequências a longo prazo}

É conhecido que experiências adversas na infância possuem repercussões que podem se prolongar na vida adulta e trazer desconforto ao indivíduo. Transtornos de ansiedade, somatização, obsessivo-compulsivos e depressivos são frequentemente observados, bem como uma grande reatividade automática e outros problemas comportamentais (ORAL R, et al., 2016).

O medo ou trauma podem desencadear um processo de adaptação envolvendo mecanismos de defesa. Sendo o indivíduo exposto a estímulos similares, por mais que estes sejam menos intensos que o original, os mesmos mecanismos são ativados.

Tal processo é conhecido como generalização do medo, e nele pode haver reações desproporcionais que causem alto prejuízo social (DUNSMOOR JE e PAZ R, 2015). Isso está relacionado ao desenvolvimento de Transtorno de Estresse Pós-Traumático (TEPT), cujas principais características consistem em reviver o evento, evitar estímulos relacionados ao trauma e aumento da excitação fisiológica (ZHAO M, et al., 2017).

Portanto, a pandemia, comportando diversos fatores geradores de estresse, como o confinamento a pequenos espaços, a falta de interação social e a ameaça do acometimento pela doença (BARBISCH D, et al., 2015), pode ser a responsável por eventos traumáticos as crianças.

A Unidade de Terapia Intensiva (UTI), quando necessária em casos graves da doença, é um ambiente que pode desencadear sensações negativas e transtornos mentais, como ansiedade, depressão e TEPT (MYHREN H, et al., 2010). Tanto o paciente quanto a família recebem inúmeros estressores, estando vulneráveis a antecipação dos desfechos acerca do estado de saúde daqueles que amam (BEESLEY SJ, et al., 2018).

As crianças com passagem pela UTI têm maiores chances de desenvolver sequelas emocionais e comportamentais, especialmente ansiedade aguda, que pode ser seguida de TEPT (SHANNON MW, et al., 2006). A crise do coronavírus, por exemplo, envolve várias características vistas em eventos traumáticos em massa, e, por isso, pode ocorrer aumento dos números de crianças e adolescentes que evoluam com TEPT durante ou após o período de pandemia, além de menor qualidade de vida e deficiências no desenvolvimento e cognição (FEGERT JM, et al., 2020).

Reportou-se, em estudo na China, que 10\% das crianças que experimentaram algum trauma em consequência da infecção pelo coronavírus podem desenvolver TEPT, catalisado pelo fato de estarem separadas dos familiares durante a internação, visto a indicação do isolamento das pessoas acometidas para evitar novas contaminações (CUI Y, et al., 2020).

A pandemia da COVID-19, pode ainda, elicitar forte medo na população em geral, além de conceber ansiedade e respostas ao estresse que podem resultar em alterações do comportamento de busca de ajuda, dificultando o cuidado e a prevenção de possíveis afecções mentais (COLIZZI M, et al., 2020). 


\section{Sensações, emoções e comportamentos experienciados ao decorrer da pandemia}

As pandemias, a exemplo da Influenza A (H1N1), são associadas ao aumento dos padrões de ansiedade e depressão, sendo possível que a crise da COVID-19 tenha impacto similar nas crianças e adolescentes. A quarentena costuma ser acompanhada de uma série de efeitos psicológicos negativos, como raiva, confusão, tédio e frustração (WITT A, et al., 2020). O estresse, um dos principais fatores observados durante estes períodos, impacta no desenvolvimento biológico da criança e do adolescente, além de produzir e reforçar comportamentos nocivos, repercutindo em toda a unidade familiar (Thompson, 2014).

A mudança no estilo de vida faz com que as pessoas tendam a se sentir ansiosas e inseguras, sendo comum encontrar, durante o isolamento social, solidão, comunicação pobre e perturbações do humor. Quanto maior for o tempo de confinamento, piores podem ser os resultados para a saúde mental, especialmente os comportamentos de evitação, raiva e ansiedade (USHER K, et al., 2020).

Verificou-se que a rápida evolução da pandemia, a obrigação da quarentena, a possibilidade de desfechos negativos e a exposição a notícias por parte da mídia, podem resultar em respostas ao estresse que podem gerar comportamentos inadequados de proteção a saúde, a exemplo da somatização (COLIZZI M, et al., 2020). Os sintomas somáticos são manifestações físicas com uma causa orgânica não identificada, culminando em perda de função. A fixação ansiosa também é um fator que pode gerar somatização (RIEM MME, et al., 2018).

Ocorreu grande prevalência do relato de insônia (CUI Y, et al., 2020; FEGERT JM, et al., 2020; SHANNON MW, et al., 2006), acompanhada de instabilidade emocional, choro frequente, depressão e medo (SHANNON MW, et al., 2006). Notificou-se que a tristeza, irritabilidade e exaustão emocional também estão presentes (FEGERT JM, et al., 2020).

\section{O impacto da família no decurso da pandemia}

É natural os pais tentarem proteger seus filhos. Em tempos de crise, manter as crianças seguras e calmas parece difícil, mas é algo que bons pais têm feito durante milhares de anos. As crianças também se preocupam com seus genitores, conseguem perceber quando estes estão conturbados e querem que eles estejam bem (NUNN K, 2020).

Há sugestões de que as crianças são mais resilientes e menos ansiosas acerca de mudanças do que seus pais (FRANIC T e DODIG-CURKOVIC K, 2020). Entretanto, estão sujeitas a riscos domésticos e a absorver pressões que a família traz (GREEN P, 2020). Os níveis de estresse familiar aumentam quando os pais são impossibilitados de trabalhar ou quando ocorre a separação destes com seus filhos devido a hospitalização (STEVENSON E, et al., 2009).

Ao estarem completamente separadas de seus pais, as crianças desenvolvem, comumente, ansiedade de separação e insônia. Quando são mantidas longe do contato presencial com amigos e professores, há maior exposição as problemáticas familiares, como o desemprego ou a recessão econômica que podem afetar os seus pais (CUI Y, et al., 2020). Atenção deve ser dada ao fato de estarem mais vulneráveis a violência doméstica, que pode ser vivenciada das mais diversas formas, tais como a violência verbal ou física, além da exposição dos mais jovens a agressões, seja na condição de expectadores ou alvos (FEGERT JM, et al., 2020).

A forma como a família reage aos acontecimentos, influencia diretamente a criança e o adolescente, podendo ser observados reflexos psicológicos negativos, como no caso do medo excessivo demonstrado por eles (SHANNON MW, et al., 2006). Os pais podem, ainda, influenciar negativamente a criança, por exemplo, ao sugerir a possibilidade de infecção da mesma ou de algum membro da família, agravando a ansiedade e os sintomas psicossomáticos (COLIZZI M, et al., 2020).

É útil fazer uso de terapias com interação pais-crianças ou psicoterapia centrada nas histórias de trauma para evitar maiores repercussões futuras (ORAL R, et al., 2016). As Práticas Integrativas e Complementares (PICs), recursos que podem vir a serem explorados, consistem em recomendações da OMS de terapias não ortodoxas que atuam na prevenção do estresse ou manifestações desencadeadas por doenças (ALMEIDA JR, et al., 2018). As PICs são vistas como potencialmente úteis em diversas situações, inclusive no tratamento complementar de sintomas orgânicos (WEEKS J, 2020). 
A fitoterapia é parte integrante das PICs, consistindo no uso de plantas medicinais em suas diferentes formas terapêuticas, sendo uma técnica de atenção a saúde, utilizada tanto na prevenção quanto no tratamento de enfermidades (COSTA IM, et al., 2019).

Muitas destas plantas são consideradas seguras e de fácil acesso, a exemplo das espécies Hypericum perforatum (erva de São João), Valeriana officinalis (valeriana) e Passiflora incarnata (maracujá), que demonstraram, em estudos clínicos já realizados, boa tolerância e mínimos efeitos colaterais (TROMPETTER I, et al., 2013).

A Matricaria recuitta chamomilla $L$. (camomila) é amplamente utilizada devido as suas propriedades ansiolíticas, antidepressivas e anti-inflamatórias, além de melhorar a qualidade do sono, tornando-se uma possibilidade versátil de fitoterápico (MIRAJ S e ALESAEIDI S, 2016).

A ampliação das opções terapêuticas ofertadas aos usuários do Sistema Único de Saúde (SUS) é uma importante estratégia, objetivando a prevenção de doenças, promoção e recuperação da saúde da população. Caminhando nesta direção, existem políticas públicas de oferta das PICs, plantas medicinais e fitoterápicos no SUS, definindo diretrizes para o acesso racional a estas terapias complementares.

Para ajudar a aliviar o estresse das crianças e adolescentes durante estes períodos, práticas como a meditação, mindfulness, exercício da espiritualidade, podem ser bastante úteis. Ao passo que o uso indevido das redes sociais e do consumo de notícias desfavoráveis podem gerar angústia. A ação dos pais de esclarecer, explicar e confortar as crianças produz calma e alivia os sentimentos negativos.

As crianças apreciam desenhar, brincar com seus pais, ler boas histórias, receber estímulos positivos e elogios. Elas também gostam de poder se comunicar com os parentes e amigos que não poderão visitar durante o período (NUNN, 2020). Estimular tais vontades pode tornar o período mais leve e confortável para os pais, crianças e adolescentes.

Mesmo na vivência da pandemia, deve-se manter o cuidado com a alimentação das crianças e adolescentes, assegurando uma rotina alimentar regular com horários pré-estabelecidos. Deve-se dar o devido destaque aos alimentos necessários para o desenvolvimento pondero-estatural normal, manutenção e fortalecimento do sistema imunológico, enfatizando os nutrientes essenciais, tais como o zinco, ferro, selênio, magnésio, vitaminas $A, E, C$ e complexo $B$.

A exposição a luz solar é, também, importante, visto que, durante o confinamento em casa, pode-se desenvolver ou agravar déficits de Vitamina D. A carência de ingestão, absorção ou aproveitamento dos mesmos pode contribuir para a deficiência da imunidade, aumentando os riscos de adoecimento e agravo de condições pré-existentes. Portanto, é prudente evitar o excesso de sódio, açúcar e gordura, mantendo a alimentação balanceada, uma boa hidratação e hábitos de higiene oral e corporal adequados.

A rotina escolar deve ser realizada em clima de tranquilidade, respeitando as orientações pedagógicas adequadas, sem maiores exigências e pressões para a família ou cobranças exageradas aos filhos (ALMEIDA RS, et al., 2020). Os pais devem estimular a prática de atividade física, mesmo que a casa tenha pouco espaço, uma vez que o sedentarismo traz diversas consequências negativas, como a ingestão excessiva de alimentos. É primordial que crianças e adolescentes tenham horas de sono suficientes, pois a exaustão devido a falta de descanso pode deixar o corpo susceptível a doenças.

É notória a necessidade de auxiliar as crianças e adolescentes nas diversas questões analisadas neste estudo, tendo em vista as consequências que o desamparo pode acarretar. Em casos de maior agitação ou sintomas ansiosos e depressivos, é importante a busca de um especialista, a exemplo dos profissionais da área de saúde mental, como médicos e psicoterapeutas.

Para tanto, observa-se o constante desenvolvimento de canais de comunicação com os profissionais de saúde, contemplando as tecnologias digitais e programas de telessaúde, com a finalidade de expandir e melhorar a rede de serviços de atenção à saúde para a população em geral. Métodos como a telepsiquiatria e atendimentos por videoconferência estão sendo utilizados com resultados satisfatórios e boa aceitação por parte dos pais que buscam ajuda para seus filhos (FRANIC T e DODIG-CURKOVIC K, 2020). 
É prudente que a família confira aos jovens a segurança, a calma e o autocuidado durante este período, enfatizando a importância da resiliência para sairmos deste momento de pandemia mais fortes e solidários. Estudos posteriores devem ser conduzidos para acompanhar o desfecho das circunstâncias atuais sobre a saúde mental da população infantojuvenil, uma vez que esta revisão ocorreu durante a pandemia da COVID19 e outras repercussões poderão ser observadas em crianças e adolescentes, com efeitos imediatos e a longo prazo.

\section{CONSIDERAÇÕES FINAIS}

Períodos de incerteza são um grande desafio para todos, especialmente para os pais e filhos, portanto, é fundamental que os pediatras e demais profissionais da saúde, estejam atentos para reconhecerem possíveis prejuízos funcionais e adotarem estratégias terapêuticas em tempo hábil, pretendendo evitar a progressão para quadros clínicos de mais difícil controle. É de suma importância fomentar políticas públicas de acolhimento, cuidado e educação, visando a prevenção de doenças e a manutenção da saúde física e mental das nossas crianças e adolescentes.

\section{AGRADECIMENTOS}

Agradecimento especial ao professor doutor Genário Alves Barbosa, psiquiatra infantil, pelo incentivo para a construção desta pesquisa e contribuição para a nossa formação acadêmica.

\section{REFERÊNCIAS}

1. AHMAD AR, et al. The Impact of Social Media on Hyped Panic during the COVID-19 Pandemic: The Iraqi Kurdistan Case. Journal of Medical Internet Research, 2020; 22(5): e19556.

2. AHN DG, et al. Current status of epidemiology, diagnosis, therapeutics, and vaccines for novel coronavirus disease 2019 (COVID-19). Journal of Microbiology and Biotechnology, 2020; 30(3): 313-324.

3. ALMEIDA JR, et al. O enfermeiro frente às práticas integrativas e complementares em saúde na

4. estratégia de saúde da família. Revista Eletrônica Acervo Saúde, 2018; 18: e77.

5. ALMEIDA RS, et al. Pandemia de COVID-19: guia prático para promoção da saúde mental de crianças e adolescentes. Residência Pediátrica, 2020; 10(2): 1-4.

6. BARBISCH D, et al. Is There a Case for Quarantine? Perspectives from SARS to Ebola. Disaster Medicine and Public Health Preparedness, 2015; 9(5): 547-553.

7. BEESLEY SJ, et al. Acute physiologic stress and subsequent anxiety among family members of ICU patients. Critical Care Medicine, 2018; 46(2): 229-235.

8. CHROUSOS GP. Stress and disorders of the stress system. Nature Reviews Endocrinology, 2009; 5(7): 374-381.

9. COLIZZI M, et al. Medically unexplained symptoms in the times of Covid-19 pandemic: a case-report. Brain, Behavior, and Immunity - Health, 2020; 5: e100073.

10. COSTA IM, et al. Uso de Plantas Medicinais e Fitoterápicos na Atenção Primária à Saúde: uma revisão integrativa. Revista Eletrônica Acervo Saúde, 2019; 26: e828.

11. CROCQ MA. A history of anxiety: From Hippocrates to DSM. Dialogues in Clinical Neuroscience, 2015; 17(3): 319325.

12. CUI Y, et al. Mental health services for children in China during the COVID-19 pandemic: results of an expert-based national survey among child and adolescent psychiatric hospitals. European Child \& Adolescent Psychiatry, 2020; 29: 743-748.

13. DUNSMOOR JE, PAZ R. Fear Generalization and Anxiety: Behavioral and Neural Mechanisms. Biological Psychiatry,2015; 78(5): 336-343.

14. EARLS F, et al. Promoting child and adolescent mental health in the context of the HIV/AIDS pandemic with a focus on sub-Saharan Africa. Journal of Child Psychology and Psychiatry and Allied Disciplines, 2008; 49(3): 295-312.

15. FEGERT JM, et al. Challenges and burden of the Coronavirus 2019 (COVID - 19) pandemic for child and adolescent mental health: a narrative review to highlight clinical and research needs in the acute phase and the long return to normality. Child and Adolescent Psychiatry and Mental Health, 2020; 14(20): 1-11.

16. FRANIC T, DODIG-CURKOVIC K. Covid-19, Child and Adolescent Mental Health - Croatian (in)experience. Irish Journal of Psychological Medicine, 2020.

17. GREEN P. Risks to children and young people during covid-19 pandemic. The British Medical Journal, 2020; 369: 12.

18. KELLY BD. Coronavirus disease: Challenges for Psychiatry. The British Journal of Psychiatry, 2020; 217(1): 1-2.

19. LIMA CKT, et al. The emotional impact of Coronavirus 2019-nCoV (new Coronavirus disease). Psychiatry Research, 2020; 287: 112915 
20. MIRAJ S, ALESAEIDI S. A systematic review study of therapeutic effects of Matricaria recuitta chamomile (chamomile). Eletronic Physician, 2016; 8(9): 3024-3031.

21. MYHREN H, et al. Posttraumatic stress, anxiety and depression symptoms in patients during the first year post intensive care unit discharge. Critical Care, 2010; 14(1): 1-10.

22. NUNN K. Keeping our children safe and calm in troubled times. Journal of Paediatrics and Child Health, 2020; 56(5): 669-671.

23. ONYEAKA HK, et al. The Unaddressed Behavioral Health Aspect During the Coronavirus Pandemic. Cureus, 2020. 12(3): 10-12.

24. ORAL R, et al. Adverse childhood experiences and trauma informed care: The future of health care. Pediatric Research, 2016; 79(2): 227-233.

25. ORNELL F, et al. "Pandemic fear" and COVID-19: mental health burden and strategies. Revista brasileira de psiquiatria, 2020; 42(3): 232-235.

26. ÖZDIN S, ÖZDIN ŞB. Levels and predictors of anxiety, depression and health anxiety during COVID-19 pandemic in Turkish society: The importance of gender. The International journal of social psychiatry, 2020.

27. PERRIN PC, et al. Preparing for an influenza pandemic: Mental health considerations. Prehospital and Disaster Medicine, 2009; 24(3): 223-230.

28. RIEM MME, et al. Attachment and medically unexplained somatic symptoms: The role of mentalization. Psychiatry Research, 2018; 268: 108-113.

29. SCHMIDT DRC, et al. Ansiedade e depressão entre profissionais de enfermagem que atuam em blocos cirúrgicos. Revista da Escola de Enfermagem da USP, 2011; 45(2): 487-493.

30. SHANNON MW, et al. Chemical-biological terrorism and its impact on children. Pediatrics, $2006 ; 118(3): 1267-1278$.

31. SHUJA KH, et al. COVID-19 Pandemic and Impending Global Mental Health Implications. Psychiatria Danubina, 2020; 32(1): 32-35.

32. STEVENSON E, et al. Pandemic influenza planning: Addressing the needs of children. American Journal of Public Health, 2009; 99(SUPPL. 2): 255-260.

33. THOMPSON RA. Stress and Child Development. The Future of Children, 2014; 24(1): 41-59.

34. TROMPETTER I, et al. Herbal triplet in treatment of nervous agitation in children. Wiener Medizinische Wochenschrift, 2013; 163(3-4): 52-57.

35. USHER K, et al. Life in the pandemic: Social isolation and mental health. Journal of clinical nursing, 2020; 29(15-16).

36. WEEKS J. Call to Action: Announcing the Traditional, Complementary and Integrative Health and Medicine COVID19 Support Registry. Journal of Alternative and Complementary Medicine, 2020; 26(4): 256-258.

37. WITT A, et al. Child and adolescent mental health service provision and research during the Covid - 19 pandemic: challenges, opportunities, and a call for submissions. Child and Adolescent Psychiatry and Mental Health, 2020; 14(19): 8-11.

38. ZHAI P, et al. The epidemiology, diagnosis and treatment of COVID-19. International Journal of Antimicrobial Agents, 2020; 55(5): 105955.

39. ZHAO M, et al. Meta-analysis of the interaction between serotonin transporter promoter variant, stress, and posttraumatic stress disorder. Scientific Reports, 2017; 7(1): 1-10 